Journal of Global Resources Volume 6 (01a) Special Issue June 2020 Page 96-101

https://doi.org/10.46587/JGR.2020.v06si01.014 ISSN: 2455-2445 (Online)

\title{
AUTOMATIC AND MULTI-DIMENSIONAL PIPE CLEANING BOT FOR COVID-19 SITUATION
}

\author{
Sudhish Subramaniam ${ }^{1}$ and Subha Subramaniam ${ }^{2}$ \\ ${ }^{1}$ Scholar, Vellore Institute of Technology, Vellore, India \\ Email: sudhish.subramaniam2018@vitstudent.ac.in \\ ${ }^{2}$ Associate Professor, Shah \& Anchor Kutchhi Engineering College (SAKEC), Mumbai, India \\ Email: subha.subramaniam@sakec.ac.in
}

\begin{abstract}
This paper focuses on developing a robot which is used to clean the inner side of pipes automatically with one operator in covid-19 situation. Cleaning inner part of pipes which carry water, gas, oil and other fluids is a challenging task and need to be addressed very effectively. Scales develop inside the pipes not only block the flow but sometimes become dangerous. Normally this process is done manually with more labors of municipal corporations. In covid-19 situation with extended lock down and less labors, pipe cleaning is considered as non-essential. We have designed a pipe cleaning robot using Arduino and ultrasonic sensor which can clean the scales and easily come out of the pipe also. This automated robot can calculate the distance inside the pipe using a sensor and can direct itself inside till the end. It has a rotor at the top which would clean the pipe with the brushes and remove all the dirt too. The uses of the bot are in such pipes where the human hand cannot reach and clean the pipes. The robot can be used to clean different diameter pipes. The shaft of the bot can be adjusted according to the diameter of the pipe. This makes the robot for cleaning multidimensional pipes in an automatic and effective way.
\end{abstract}

Key words: Scales, Diameter, Microcontroller, Sensor

\section{Introduction}

A pipe is a tube used to convey water, gas, oil, or other fluid substances. These pipes have been used in our daily lives to the maximum. Pipes are used to transport so many essential things from one place to the other throughout the day, night and have to face so many weather conditions like rain, winter and summer conditions. These conditions not only affect the efficiency of the pipes but also restrict the flow of the substances inside. Scales are some materials which generate inside the pipes. These materials are formed due to the minerals present in the liquid or substance flowing inside the pipe as in fig.1. In water this essentially means Calcium and Magnesium. As water flows, small irregularities in the pipe's surface will catch impurities. These impurities will continue to catch on these rough spots, causing them to grow. These Scales sometimes become very dangerous and tend to block the flow of the liquid inside the pipe. With the non-uniformity of the surface of the pipe the formation of scales increases and hence increases the formation of scales on the surface. The formation of scales increases to the top and after a period of time starts pilling up which increases the pressure inside the pipe and hence blocks the flow. In some severe cases these blocks become very tedious and lead to bursting of the pipe. This happens in cases where the pipe cannot handle the pressure of the liquid flow due to the blockage.

The optimum way is to prevent the formation of such scales on the inner surface of the pipes. One of the best ways to prevent the formation of the scales is regular scrubbing which smoothens the inner surface of the pipes and remove the loose scales formed on the surface. Some method such as high power blowing also removes the scales but we find this as a better way to remove the scales. The project aims to build a robot that would surely scrub the inner parts of 
the pipe and hence decrease the formation of scales inside the pipe. It would be an automated bot that would calculate the distance inside the pipe using a sensor and would direct itself inside till the end. The bot would have rotor at the top which would clean the pipe with the brushes and remove all the dirt too. This bot would be designed in such a manner that it clears the whole pipe and automatically come out. The uses of the bot are in such pipes where the human hand cannot reach and clean the pipes. Such bots will surely be very helpful to clean the whole pipe and preventing the formation of scales. Our bot is designed in such a way that it enters the pipe calculates the length of it and scrubs the whole pipe from inside and collects all the dirt settled at the bottom and exits the pipe.

\section{Literature Review}

There are some systems available, wherein people have tried to implement a similar kind of system they are as follows: In [1] Ana Sakura et.al created a developed a cleaning device for inpipe robot application. Their device is able to clean the pipes moderately. The device is suitable for routine cleaning process. In [2] Abdullah Al Deghreer et.al created a pipe inspection and cleaning robot. It is a robot that will be used to clean the interior of the pipes using a brushing mechanism. One of the critical areas of the oil and gas industry is the transport of oil and other fluids through a network of pipes. Over time these pipes have accumulated amount of slug and other deposits; this leads to decrease in pipeline carrying capacity, reduced reliability, loss of power due to higher pumping pressure required and irregular flow are achieved which in a nutshell was to build a prototype PIAC with the mobility, cleaning and video feedback functionality.

\section{Overview of Proposed System}

To develop an automated, multi-dimensional BOT to clean the scales in the inner areas of pipes carrying water, liquid, gas and any other fluid. The Bot is completely automated it functions according the value of the Ultrasonic Sensor. According to the value of the ultrasonic sensor, the bot is designed to operate in the forward or backward motion. As soon as the end of the pipe is detected by the ultrasonic sensor the bot is designed in such a way that it performs the following functions: - The PWM of the pipe is changed and the brushes are rotated by an angle of 45 degree in clockwise direction. Then using the motor driver, the polarity of the DC motor is changed such that the bot runs in the opposite direction. The opening of the pipe has to be closed when the bot is sent inside. The Bot then detects the closing and rotates the brushes in anticlockwise direction by changing the polarity changes the polarity. When one half cycle of the brush is completed the bot beeps a sound using a buzzer and the closing Has to be opened. This type of positioning of the brushes helps in removing the scales which will be settled down after every rotation. Scales formed inside a pipe which causes obstruction in flow or blockage.

Hardware Requirements

Components used:

- Arduino UNO

- Ultrasonic Sensor

- DC motors

- Servo motor

- Motor Driver

- Mechanical Set 
Figure 01: Scales formed inside the Pipe

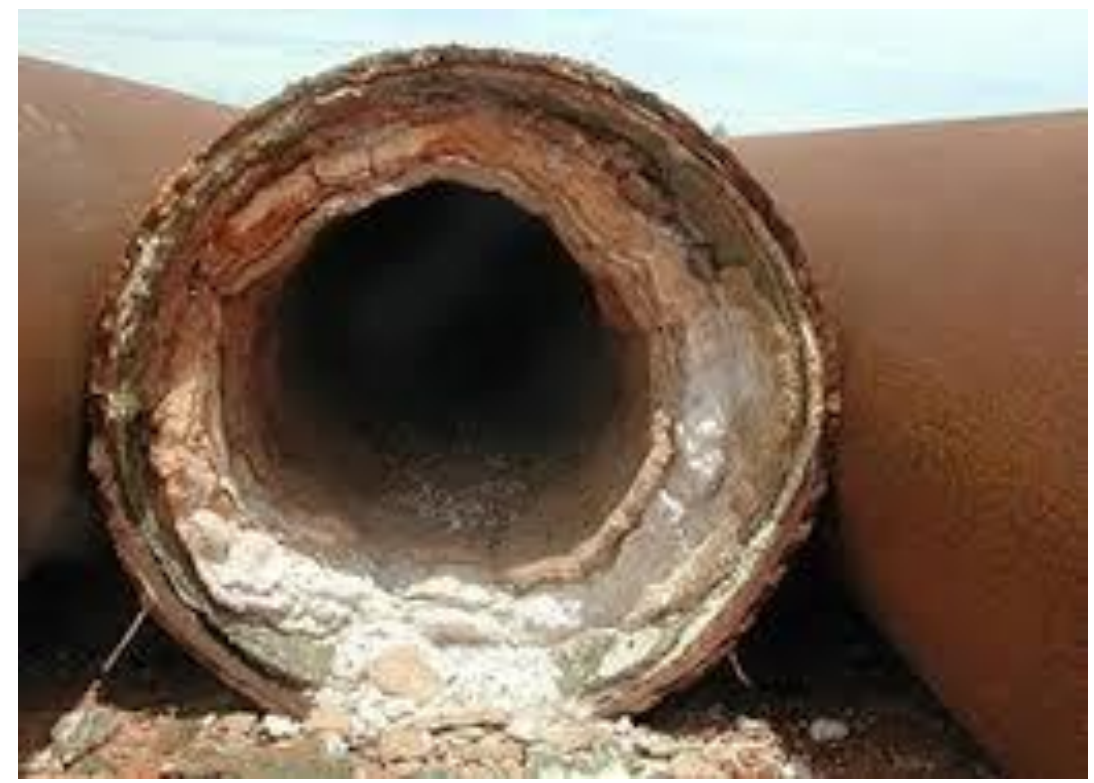

\section{Working of the Ultrasonic Sensor}

Ultrasonic sensor: Ultrasonic sensor works as per the given fig.2. This sensor uses the crystal oscillator to generate ultrasonic waves which is sent and received by the transmitter and receiver. The time in which the sensor again senses the ultrasonic waves tells the distance between the sensor and the object. As per the difference in sending and receiving of data, the sensor gives the distance of the obstacle.

Figure 02: Working of Ultrasonic Sensor

\section{WORKING}

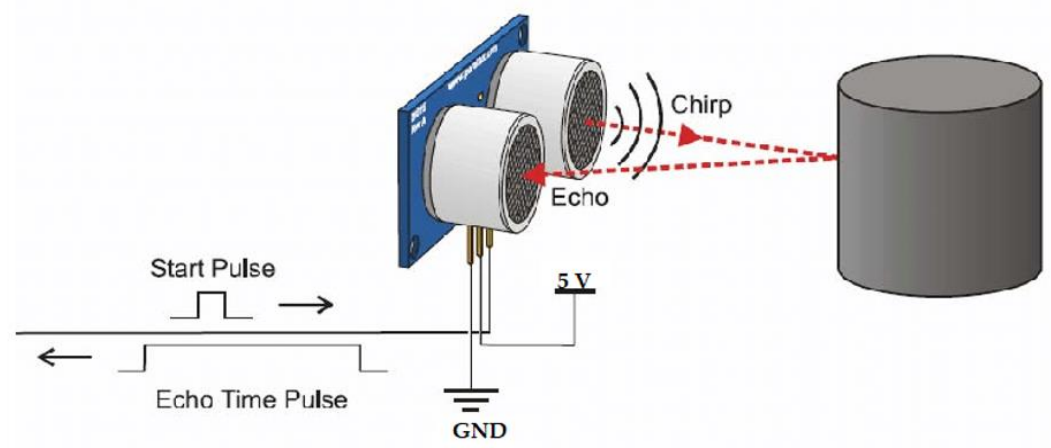

Software Requirements

- Arduino IDE for programming.

- Proteus Software for circuit Designing

- Express PCB for PCB designing

\section{Design Details}
1) BLOCK DIAGRAM 
Figure 03: Block Diagram of the BOT Working

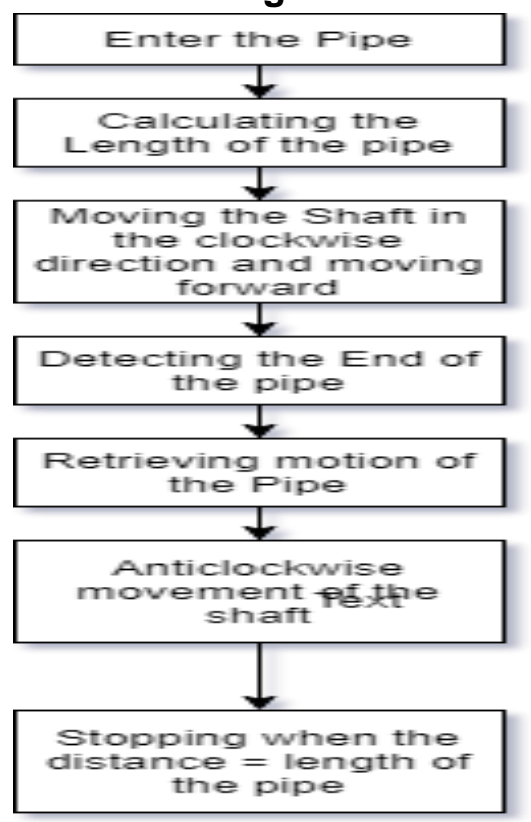

\section{Working of Pipe Cleaning BOT}

As in fig. 3 we can see that the BOT enters the pipe and initially calculate the length of the pipe. It moves shaft in clockwise direction and moves forward. It keeps moving and detects the end of the pipe. Once it detects the end of the pipe it retrieves its path. The BOT initiates the anticlockwise movement of the shaft. The above procedure is the developed BOT algorithm for this pipe cleaning BOT. The clockwise motion of the shaft helps in scrubbing the pipe once and hence the all the dust and scales are settled at the bottom of the pipe. Once the bot reaches the end of the pipe, the anticlockwise motion of the shaft pulls out all the dust and the scales out of the pipe. This BOT algorithm is explained in Fig.6 and well explained in the flowchart. The BOT stops when the distance is equal to the length of the pipe.

Figure 04: Flowchart of BOT Working

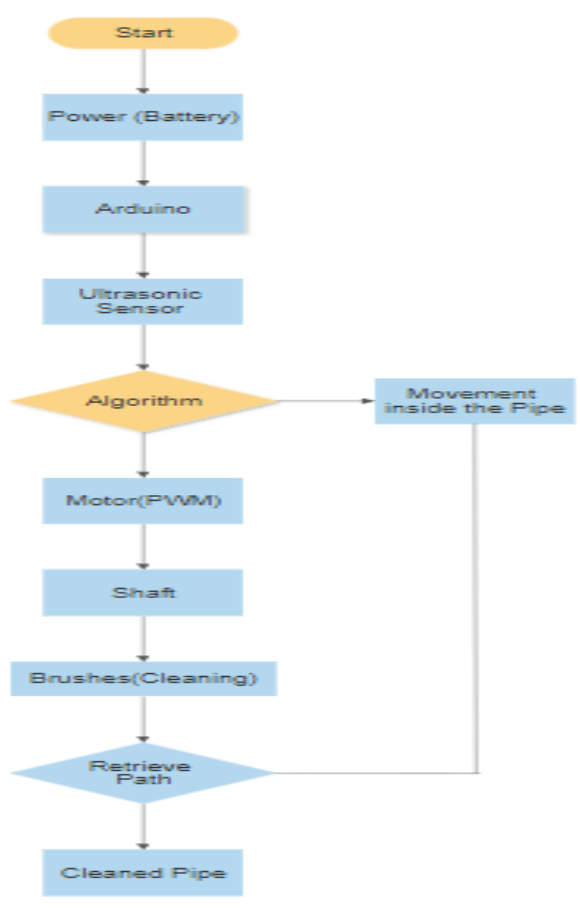


The flowchart shown in Figure 4 depicts the entire working of the pipe cleaning BOT. As soon as the BOT is powered, Arduino and ultra-sonic sensor gets power start sensing the distance. As per the BOT algorithm the length of pipe is calculated. The brushes are connected at the shaft of Arduino. It rotates the brush clockwise till the end of pipe. By the rotation of shaft and brushes the scales are cleaned. The movement of the BOT inside the pipe is completely controlled by the BOT algorithm and thus making the BOT completely automatic. The BOT can be used to clean different diameter pipes. The shaft of the bot can be adjusted according to the diameter of the pipe. The BOT is made for cleaning different radius pipes in an automatic and effective way. The Fig.5.1, Fig.5.2, Fig.5.3 depicts the structure of the BOT. Fig.6 shows the algorithm of the BOT's code and depicts the working.

\section{IMAGES OF THE BOT}

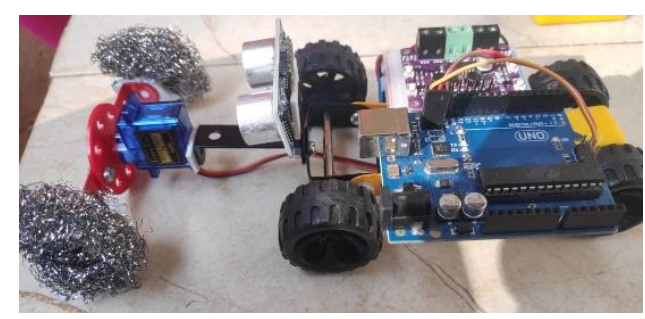

Figurev05.1: Side View of the BOT

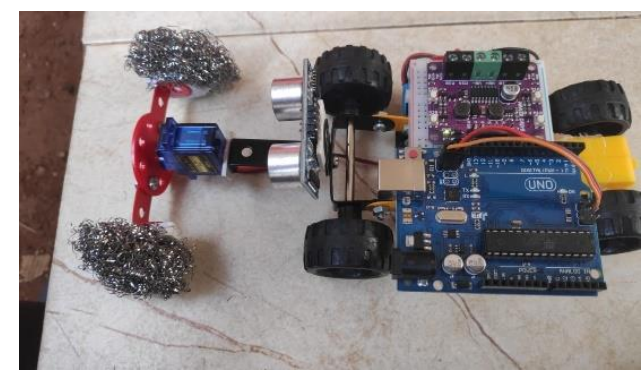

Figure 5.2: Top View of the BOT

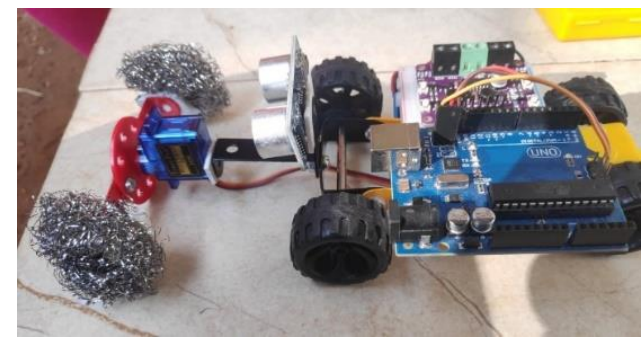

Figure 5.3: BOT Structure

\section{Advantages}

- Completely automatic; No manual help is needed

- Many Pipes are not accessible by human hand and are difficult to clean by bare hands.

- Multi-dimensional pipes can be cleaned

- Highly useful for pipe cleaning in urban location.

- After implementation maintenance of this system is very low.

- This BOT system can be used in metropolitan cities like Mumbai where population density is more.

- During pandemic situations like COVID-19, instead of manual cleaning, BOT can be used as automatic cleaning system. This can save life of humans.

- A UNIQUE BOT algorithm is developed for shaft movement. 


\section{Conclusion}

In this situation, we were able to develop an automatic, multidimensional pipe cleaning BOT in pandemic COVID 19 situation. Human lives are affected hugely by COVID 19 virus in this year 2020. Social distancing is more important and lives of Municipal Corporation staff are at high risk. As a solution to this pandemic situation, the entire country is in lock down stage for more than 2 months. In the coming years also it is a great challenge to maintain clean environment and social distancing. It is very difficult to clean pipes which supply water to cities. This pandemic situation has forced us to be inside are homes, but we are able to get resources only because of the people at the municipal cooperation who maintain the pipes in this dangerous situation. So much of man power is required and it is done manually in India till today. This BOT can solve many above mentioned issues and provide a clean pipe. Keeping their life in mind and the pandemic situation this bot will surely help to reduce the pipe pressure caused due to scales and would also save the lives of the municipal workers and ease the situation to a great extent. Being multi-dimensional the BOT shaft can be adjusted according to the diameter of the pipes. It further saves human lives and help in clean and safe environment.

\section{Figure 06: Flowchart of Algorithm of the BOT}

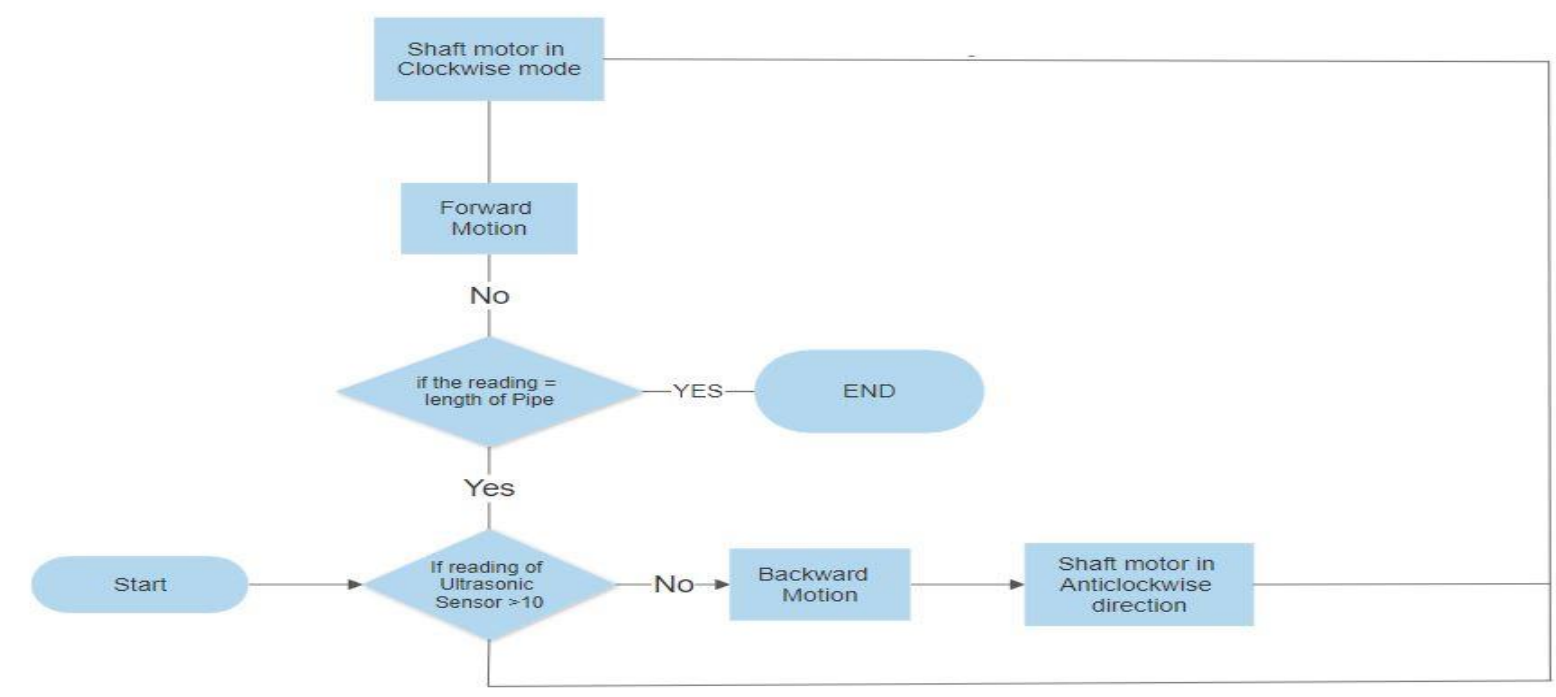

\section{References}

1. Abdullah Al Deghreer, Mohammed Alyami, Naif Al Mahasheer, Ahmed Al Shalawi, (2016). “ Pipe Inspection and Cleaning Robot", project report, Prince Mohammad Bin Fahd University,

2. Ana Sakura Zainal Abidin, Annisa Jamal, Shahrol Mohamaddan, (2015). "Development of Cleaning Device for In-pipe Robot Application", ScienceDirect, Procedia Computer Science 76 506 - 511, Elsevier, 2015 IEEE International Symposium on Robotics and Intelligent Sensors (IRIS 2015)

3. Collins M., Drain valve and pipe blockage clearing device, (1999). D.S.J.D. Ellison, Investigation of Pipe Cleaning Method, pp. 91Y92, 2003.

4. Enner F., Rollinson D. and Choset H., (2013). Motion Estimation of snaNe robots in straight Pipes, IEEE International conference on robotics and automation, pp. 5168Y5173.

5. M.G. A. L.L.J. Gagliardi, L. (2000). Water systems piping," Piping handbook, vol. part c, no. Piping system, 